PROCEEDINGS OF THE

AMERICAN MATHEMATICAL SOCIETY

Volume 141, Number 11, November 2013, Pages 4039-4048

S 0002-9939(2013)11667-9

Article electronically published on July 18, 2013

\title{
HOFBAUER TOWERS AND INVERSE LIMIT SPACES
}

\author{
LORI ALVIN \\ (Communicated by Bryna Kra)
}

\begin{abstract}
In this paper we use Hofbauer towers for unimodal maps to study the collection of endpoints of the associated inverse limit spaces. It is shown that if $f$ is a unimodal map for which the kneading map $Q_{f}(k)$ tends to infinity and $\left.f\right|_{\omega(c)}$ is one-to-one, then the collection of endpoints of $(I, f)$ is precisely the set $\mathcal{E}_{f}=\left\{\left(x_{0}, x_{1}, \ldots\right) \in(I, f) \mid x_{i} \in \omega(c)\right.$ for all $\left.i \in \mathbb{N}\right\}$.
\end{abstract}

\section{INTRODUCTION}

There has been a great deal of interest in understanding the topological structure of an inverse limit space with a single bonding map (see, for example, 11, 2, 4, 7]). One of the motivating questions in studying inverse limit spaces is Ingram's Conjecture: given two distinct symmetric tent maps $f$ and $g$, the inverse limit spaces $(I, f)$ and $(I, g)$ are not homeomorphic. This conjecture was recently proven in the affirmative by Barge, Bruin and Stimac [3]; however, the topological structure of these inverse limit spaces is still not completely understood, making it difficult to actually distinguish between inverse limit spaces.

One key to understanding the topological structure of an inverse limit space is to better understand its collection of endpoints, as endpoints are a topological invariant. In [4 Barge and Martin provide a characterization for a point to be an endpoint of an inverse limit space in the case where the bonding map is continuous over an interval; more recently there have been several additional characterizations for different classes of bonding maps. Bruin provides a characterization in the case where the bonding map is unimodal with a non-periodic turning point [7]. More recently Alvin and Brucks modified Bruin's result when the single bonding map is unimodal with an embedded adding machine [1].

We are interested in precisely defining the collection of endpoints for a given inverse limit space $(I, f)$, where $f$ is unimodal. By further understanding the collection of endpoints and how they behave within the inverse limit space, we hope to better understand the topological structure of the inverse limit space. We also hope to use invariant structures such as the collection of endpoints to help us distinguish between two inverse limit spaces.

It is known that every endpoint of $(I, f)$ lies in $\mathcal{E}_{f}=\left\{\left(x_{0}, x_{1}, \ldots\right) \in(I, f) \mid\right.$ $x_{i} \in \omega(c)$ for all $i \in \mathbb{N}$ \} (see, for example, [1]). In [2] the relationship between the inverse limit space $(I, f)$ and the behavior of the kneading map $Q_{f}(k)$ was explored. It was shown that every unimodal map $f$ with $\lim _{k \rightarrow \infty} Q_{f}(k)=\infty$ and $\left.f\right|_{\omega(c)}$ topologically conjugate to an adding machine is such that $\mathcal{E}_{f}$ is precisely the

Received by the editors October 19, 2011 and, in revised form, January 23, 2012.

2010 Mathematics Subject Classification. Primary 54H20, 37B45; Secondary 37E05.

Key words and phrases. Hofbauer tower, inverse limit spaces, kneading maps, endpoints. 
collection of endpoints of $(I, f)$. It was further shown that there exist examples of unimodal maps $f$ with embedded adding machines and $\lim _{k \rightarrow \infty} Q_{f}(k) \neq \infty$ where the collection of endpoints of $(I, f)$ is exactly $\mathcal{E}_{f}$ and examples where the endpoints are properly contained in $\mathcal{E}_{f}$. We note that if $f$ has an embedded adding machine, then $\left.f\right|_{\omega(c)}$ is one-to-one. In this paper we generalize our results from [2] to show that if $f$ is a unimodal map with $\lim _{k \rightarrow \infty} Q_{f}(k)=\infty$ and $\left.f\right|_{\omega(c)}$ is one-to-one, then the collection of endpoints of $(I, f)$ is precisely $\mathcal{E}_{f}$. It is still unknown if every unimodal map $f$ with $\lim _{k \rightarrow \infty} Q_{f}(k)=\infty$ is such that $\mathcal{E}_{f}$ is the collection of endpoints of $(I, f)$.

In Section 2 we provide an overview of the definitions and preliminary results that are used throughout this paper. We briefly recall several known results about endpoints of inverse limit spaces in Section 2.3. In Section 3 we review the concept of the Hofbauer tower for a unimodal map; several results about the Hofbauer tower are verified that will be heavily utilized in Section 4. In Theorem 4.1 we prove that if $f$ is a unimodal map, $\lim _{k \rightarrow \infty} Q_{f}(k)=\infty$, and $\left.f\right|_{\omega(c)}$ is one-to-one, then $\mathcal{E}_{f}$ is precisely the collection of endpoints of $(I, f)$. We then define nearly one-to-one on an omega-limit set and extend Theorem 4.1 to hold when $\left.f\right|_{\omega(c)}$ is nearly one-toone. We conclude this paper by making a few observations about the set $\mathcal{E}_{f}$ that can be used in some cases to clearly distinguish between two inverse limit spaces.

\section{BACKGROUND}

In this section we establish the terminology and preliminary results that will be used throughout this paper. We begin by reviewing unimodal maps and some associated definitions and properties. We then recall the concept of an adding machine before referencing several results about inverse limit spaces that will be expanded upon in Section 4 .

2.1. Unimodal maps. A unimodal map is a continuous map $f:[0,1] \rightarrow[0,1]$ for which there exists a point $c \in(0,1)$ such that $\left.f\right|_{[0, c]}$ is strictly increasing and $\left.f\right|_{[c, 1]}$ is strictly decreasing. This point $c$ is called the turning point, and for all $i \in \mathbb{N}$ we set $c_{i}=f^{i}(c)$. Two common families of unimodal maps are the symmetric tent family and the logistic family. A symmetric tent map $T_{a}:[0,1] \rightarrow[0,1]$ with $a \in[0,2]$ is given by

$$
T_{a}(x)= \begin{cases}a x & \text { if } x \leq \frac{1}{2}, \\ a(1-x) & \text { if } x \geq \frac{1}{2} .\end{cases}
$$

The logistic map $g_{a}:[0,1] \rightarrow[0,1]$ with $a \in[0,4]$ is defined by $g_{a}(x)=a x(1-x)$.

For the remainder of this paper, we assume $f$ is a unimodal map with $c_{2}<c<c_{1}$ and $c_{2} \leq c_{3}$. Then the interval $\left[c_{2}, c_{1}\right]$, called the core of $f$, is invariant. Let $f^{n}$ be an iterate of $f$ and $J$ be a maximal subinterval for which $c \in \partial J$ and $\left.f^{n}\right|_{J}$ is monotone; then $f^{n}: J \rightarrow[0,1]$ is called a central branch. An iterate $n$ is called a cutting time if the image of the central branch of $f^{n}$ contains $c$. The cutting times are denoted $S_{0}, S_{1}, S_{2}, \ldots$, where $S_{0}=1$ and $S_{1}=2$. The difference between two consecutive cutting times is again a cutting time, so we may define an integer function $Q_{f}: \mathbb{N} \rightarrow \mathbb{N} \cup\{0\}$, called the kneading map, by $S_{k}-S_{k-1}=S_{Q_{f}(k)}$; we note that not every integer function is a kneading map [11]. Given a sequence of cutting times or a kneading map, the associated symmetric tent or logistic map may be completely determined. 
Given a unimodal map $f$ and $x \in[0,1]$, the itinerary of $x$ under $f$ is given by $I(x)=I_{0} I_{1} I_{2} \cdots$, where $I_{j}=1$ if $f^{j}(x)>c, I_{j}=0$ if $f^{j}(x)<c$, and $I_{j}=*$ if $f^{j}(x)=c$. We call $I_{j}$ the address of the point $f^{j}(x)$ and make the convention that the itinerary stops after the first $*$ appears. The kneading sequence of a map $f$, denoted $\mathcal{K}(f)$, is the sequence $I(f(c))$. For ease of notation we write $\mathcal{K}(f)=e_{1} e_{2} e_{3} \cdots$; that is, $e_{i}$ denotes the address of $c_{i}$. If there exists some $i \in \mathbb{N}$ such that $e_{i}=*$, then we say $c$ is periodic and the kneading sequence is finite; otherwise $\mathcal{K}(f)$ is infinite.

Given a finite sequence $v$ of 0 's and 1's, we say $v$ has even parity if an even number of 1's appears in $v$; otherwise $v$ has odd parity. One puts the parity-lexicographical ordering (plo for short) on itineraries. The plo is a slight variation on the usual lexicographical ordering and works as follows: Suppose $v \neq w$ are itineraries. Find the first position where $v$ and $w$ differ, then compare in that position using the ordering $0 \prec * \prec 1$ if the number of 1 's preceding this position is even and use the ordering $0 \succ * \succ 1$ otherwise. This ordering is motivated by the property that for all unimodal maps $f$ and for all $x, y \in[0,1], I(x) \preceq I(y)$ if and only if $x<y$ (see, for example, [9, Section II.1]).

A unimodal map $f$ (with turning point $c$ ) is renormalizable of period $n \geq 2$ provided there exists an interval $J \ni c$ such that $f^{n}(J) \subset J$ and $\left.f^{n}\right|_{J}$ is again unimodal; such an interval is called restrictive. If we repeat this process infinitely often, we say the map $f$ is infinitely renormalizable. We note that every tent map $T_{a}$ with $a \in(1, \sqrt{2}]$ is finitely renormalizable and every tent map $T_{a}$ with $a \in(\sqrt{2}, 2]$ is non-renormalizable; no map in the tent family is infinitely renormalizable. Throughout this paper we assume all unimodal maps have no wandering intervals and no attracting periodic orbits. Hence, if the unimodal map $f$ is not renormalizable, we may assume $f$ is from the symmetric tent family. In the case where $f$ is renormalizable, we may take $f$ to be a logistic map [6, Chapter 3].

Recall that the omega-limit set of a point $x \in[0,1]$ is defined by $\omega(x, f)=$ $\omega(x)=\left\{y \in[0,1] \mid\right.$ there exists $n_{1}<n_{2}<\cdots$ with $\left.f^{n_{i}}(x) \rightarrow y\right\}$. There are many known connections between the behavior of the kneading map $Q_{f}(k)$ and the set $\omega(c)$.

Lemma 2.1 ([8, Lemma 2.1]). Let $f$ be a unimodal map (with no wandering intervals and no attracting periodic orbits) and suppose $\lim _{k \rightarrow \infty} Q_{f}(k)=\infty$. Then $\omega(c)$ is a minimal Cantor set.

2.2. Adding machines. The results in [1] and [2], which preceded this work, focus on inverse limit spaces where the single bonding map has an embedded adding machine.

Let $\alpha=\left\langle q_{1}, q_{2}, \ldots\right\rangle$ be a sequence of integers where each $q_{i} \geq 2$. Define $\Delta_{\alpha}$ to be the set of all sequences $\left(a_{1}, a_{2} \ldots\right)$ such that $0 \leq a_{i} \leq q_{i}-1$ for each $i$. Apply the metric $d_{\alpha}$ to $\Delta_{\alpha}$ by

$$
d_{\alpha}\left(\left(x_{1}, x_{2}, \ldots\right),\left(y_{1}, y_{2}, \ldots\right)\right)=\sum_{i=1}^{\infty} \frac{\delta\left(x_{i}, y_{i}\right)}{2^{i}},
$$

where $\delta\left(x_{i}, y_{i}\right)=0$ if $x_{i}=y_{i}$ and $\delta\left(x_{i}, y_{i}\right)=1$ otherwise. Addition on $\Delta_{\alpha}$ is defined as follows. Set

$$
\left(x_{1}, x_{2}, \ldots\right)+\left(y_{1}, y_{2}, \ldots\right)=\left(z_{1}, z_{2}, \ldots\right)
$$


where $z_{1}=\left(x_{1}+y_{1}\right) \bmod q_{1}$, and for each $j \geq 2, z_{j}=\left(x_{j}+y_{j}+r_{j-1}\right) \bmod q_{j}$ with $r_{j-1}=0$ if $x_{j-1}+y_{j-1}+r_{j-2}<q_{j-1}$ and $r_{j-1}=1$ otherwise (we set $r_{0}=0$ ). Define $f_{\alpha}: \Delta_{\alpha} \rightarrow \Delta_{\alpha}$ by

$$
f_{\alpha}\left(\left(x_{1}, x_{2}, \ldots\right)\right)=\left(x_{1}, x_{2}, x_{3}, \ldots\right)+(1,0,0, \ldots) .
$$

The dynamical system $f_{\alpha}: \Delta_{\alpha} \rightarrow \Delta_{\alpha}$ is the $\alpha$-adic adding machine map. We note that $f_{\alpha}$ is one-to-one and onto.

It is well-known that if $f$ is an infinitely renormalizable unimodal map, then $f$ has an embedded adding machine [10, Proposition III.4.5]. Further, it was shown in [5] that adding machines can be embedded in non-infinitely renormalizable unimodal maps; these adding machines are called strange adding machines.

We set $\mathcal{A}$ to be the collection of unimodal maps whose restriction to the omegalimit set of the turning point is topologically conjugate to an adding machine. In this work we investigate the collection of endpoints for an inverse limit space when the single bonding map is such that $\left.f\right|_{\omega(c)}$ is one-to-one, a weaker condition than $f \in \mathcal{A}$.

2.3. Inverse limit spaces and endpoints. Given a continuum $I$ (i.e., a compact connected metric space) and a continuous map $f: I \rightarrow I$, the associated inverse limit space $(I, f)$ is defined by

$$
(I, f)=\left\{x=\left(x_{0}, x_{1}, \ldots\right) \mid x_{n} \in I \text { and } f\left(x_{n+1}\right)=x_{n} \text { for all } n \in \mathbb{N}\right\}
$$

and has metric

$$
d(x, y)=\sum_{i=0}^{\infty} \frac{\left|x_{i}-y_{i}\right|}{2^{i}} .
$$

The map $\hat{f}:(I, f) \rightarrow(I, f)$ given by $\hat{f}\left(\left(x_{0}, x_{1}, \ldots\right)\right)=\left(f\left(x_{0}\right), x_{0}, x_{1}, \ldots\right)$ is called the induced homeomorphism on $(I, f)$. For $x \in(I, f)$, the $i$ th projection of $x$ is denoted by $\pi_{i}(x)=x_{i}$. The backward itinerary of a point $x \in(I, f)$ is defined coordinate-wise by $\mathcal{I}_{i}(x)$, where $\mathcal{I}_{i}(x)=1$ if $x_{i}>c, \mathcal{I}_{i}(x)=0$ if $x_{i}<c$, and $\mathcal{I}_{i}(x)=*$ if $x_{i}=c$.

As in 7, for each $x \in(I, f)$ such that $x_{i} \neq c$ for all $i>0$, set

$$
\begin{gathered}
\tau_{R}(x)=\sup \left\{n \geq 1 \mid \mathcal{I}_{n-1}(x) \mathcal{I}_{n-2}(x) \cdots \mathcal{I}_{1}(x)=e_{1} e_{2} \cdots e_{n-1}\right. \text { and } \\
\left.e_{1} e_{2} \cdots e_{n-1} \text { has even parity }\right\}
\end{gathered}
$$

and

$$
\begin{gathered}
\tau_{L}(x)=\sup \left\{n \geq 1 \mid \mathcal{I}_{n-1}(x) \mathcal{I}_{n-2}(x) \cdots \mathcal{I}_{1}(x)=e_{1} e_{2} \cdots e_{n-1}\right. \text { and } \\
\left.e_{1} e_{2} \cdots e_{n-1} \text { has odd parity }\right\} .
\end{gathered}
$$

Note that in general, $\tau_{L}(x)$ and/or $\tau_{R}(x)$ can be infinite. Further, for each $x \in$ $(I, f)$, we set $\Gamma(x)=\left\{y \in(I, f) \mid \mathcal{I}_{i}(y)=\mathcal{I}_{i}(x)\right.$ for all $\left.i \geq 1\right\}$.

As we focus on unimodal bonding maps, our inverse limit spaces are atriodic (i.e., contain no homeomorphic copies of the letter $Y$ ); hence we may use the following definition. A point $x \in(I, f)$ is an endpoint of $(I, f)$ provided that for every pair $A$ and $B$ of subcontinua of $(I, f)$ with $x \in A \cap B$, either $A \subset B$ or $B \subset A$. Throughout the remainder of the paper, when discussing inverse limit spaces, $I$ denotes the core $\left[c_{2}, c_{1}\right]$ of a given unimodal map.

In [7] Bruin proves the following two results. Proposition 2.2 gives combinatoric and analytic tools that can be used to characterize endpoints in an inverse limit space. Namely, the backward itinerary of the point $x \in(I, f)$ must agree for an arbitrarily long time with the kneading sequence $\mathcal{K}(f)$ and the $x_{0}$-coordinate must 
be precisely identified as either $\sup \pi_{0}(\Gamma(x))$ or inf $\pi_{0}(\Gamma(x))$. Lemma 2.3 aids us in further identifying sup $\pi_{0}(\Gamma(x))$ and inf $\pi_{0}(\Gamma(x))$ in terms of the iterates of the turning point of $f$. Both Proposition 2.2 and Lemma 2.3 will be used to identify the endpoints for our unimodal maps in Section 4. That is, there exists a strong connection between the kneading sequence $\mathcal{K}(f)$ and the collection of endpoints of $(I, f)$.

Proposition 2.2 ([7, Proposition 2]). Let $f$ be a unimodal map for which $c$ is nonperiodic and suppose $x=\left(x_{0}, x_{1}, x_{2}, \ldots\right) \in(I, f)$ is such that $x_{i} \neq c$ for all $i \geq 0$. Then $x$ is an endpoint of $(I, f)$ if and only if $\tau_{R}(x)=\infty$ and $x_{0}=\sup \pi_{0}(\Gamma(x))$ (or $\tau_{L}(x)=\infty$ and $x_{0}=\inf \pi_{0}(\Gamma(x))$ ).

Lemma 2.3 ([7, Lemma 3]). If $x \in(I, f)$, then

$$
\begin{aligned}
& \sup \pi_{0}(\Gamma(x))=\inf \left\{c_{n} \mid \mathcal{I}_{n-1}(x) \mathcal{I}_{n-2}(x)\right. \cdots \mathcal{I}_{1}(x)=e_{1} e_{2} \cdots e_{n-1} \text { and } \\
&\left.e_{1} e_{2} \cdots e_{n-1} \text { has even parity }\right\}
\end{aligned}
$$

and

$$
\begin{aligned}
\inf \pi_{0}(\Gamma(x))=\sup \left\{c_{n} \mid \mathcal{I}_{n-1}(x) \mathcal{I}_{n-2}(x) \cdots \mathcal{I}_{1}(x)\right. & =e_{1} e_{2} \cdots e_{n-1} \text { and } \\
& \left.e_{1} e_{2} \cdots e_{n-1} \text { has odd parity }\right\} .
\end{aligned}
$$

For a unimodal map $f$, we define $\mathcal{E}_{f}:=\left\{\left(x_{0}, x_{1}, \ldots\right) \in(I, f) \mid x_{i} \in \omega(c)\right.$ for all $i \in \mathbb{N}\}$. When the map $f$ is clearly understood in the context, we simply denote this set by $\mathcal{E}$. We now recall some known results about the relationship between $\mathcal{E}$ and the collection of endpoints of $(I, f)$. The next lemma also follows from 4 and [7.

Lemma 2.4 ([1, Lemma 3.2]). Let $f$ be a unimodal map with $\mathcal{K}(f) \neq 10^{\infty}$ and suppose $x=\left(x_{0}, x_{1}, \ldots\right) \in(I, f) \backslash \mathcal{E}$. Then $x$ is not an endpoint of $(I, f)$.

Note that in applying Proposition [2.2, it suffices to check only the points $x=$ $\left(x_{0}, x_{1}, x_{2}, \ldots\right) \in \mathcal{E}$. Further, as $c$ is assumed to be non-periodic, $x_{i}=c$ for at most one $i \in \mathbb{N}$. If this is the case, we set $y=\hat{f}^{-(i+1)}(x)$ and note that $x$ is an endpoint of $(I, f)$ if and only if $y$ is an endpoint of $(I, f)$. As $y_{i} \neq c$ for all $i \geq 0$, we may check whether $y$ is an endpoint using Proposition 2.2 .

The following is a modification of Proposition 2.2 in the case where the unimodal map $f \in \mathcal{A}$.

Theorem 2.5 ([1, Theorem 3.4]). Let $f \in \mathcal{A}$ and $x \in \mathcal{E}$ be such that $x_{i} \neq c$ for all $i \geq 0$. Then $x$ is an endpoint of $(I, f)$ if and only if $\tau_{R}(x)=\infty$ or $\tau_{L}(x)=\infty$.

Corollary 2.6 ([1, Corollary 3.6]). Let $f$ be an infinitely renormalizable logistic map. Then $\mathcal{E}$ is precisely the collection of endpoints of $(I, f)$.

Note that if $f$ is infinitely renormalizable, then $\lim _{k \rightarrow \infty} Q_{f}(K)=\infty$. In [1] it is also shown that if $f$ is a tent map with an embedded strange adding machine constructed as in the proof of [5, Theorem 3.1], then the collection of endpoints of $(I, f)$ is a proper subset of $\mathcal{E}$; in this case $\lim _{k \rightarrow \infty} Q_{f}(k) \neq \infty$. In [2] it is shown that there do exist examples of strange adding machines embedded in unimodal maps $f$ for which $\lim _{k \rightarrow \infty} Q_{f}(k) \neq \infty$ and the collection of endpoints of $(I, f)$ is precisely $\mathcal{E}$. Further, if $f \in \mathcal{A}$ and $\lim _{k \rightarrow \infty} Q_{f}(k)=\infty$, then it is always the case that the collection of endpoints of $(I, f)$ is exactly $\mathcal{E}[2$. In this paper we extend 
these results to show that if $f$ is a unimodal map with $\lim _{k \rightarrow \infty} Q_{f}(k)=\infty$ and $\left.f\right|_{\omega(c)}$ is one-to-one, then the collection of endpoints of $(I, f)$ is exactly $\mathcal{E}$. We now review Hofbauer towers, as they will be heavily utilized in proving the main results of this paper.

\section{Hofbauer towers}

One can combinatorically characterize certain dynamical behaviors of unimodal maps using both Hofbauer towers and kneading maps. For example, the orbit of the turning point can be easily followed in the construction of the Hofbauer tower.

Given a unimodal map $f$, the associated Hofbauer tower is the disjoint union of intervals $\left\{D_{n}\right\}_{n \geq 1}$ where $D_{1}=\left[0, c_{1}\right]$ and, for $n \geq 1$,

$$
D_{n+1}= \begin{cases}f\left(D_{n}\right) & \text { if } c \notin D_{n}, \\ {\left[c_{n+1}, c_{1}\right]} & \text { if } c \in D_{n} .\end{cases}
$$

We denote the interval $D_{n}=\left[c_{n} ; c_{\beta(n)}\right]$; this notation with the semicolon represents the fact that the order of the endpoints for this closed interval is unknown, and it may be that either $c_{n}<c_{\beta(n)}$ or $c_{\beta(n)}<c_{n}$. Here $\beta(n)=n-S_{k}$, where $S_{k}$ is the largest cutting time less than $n$.

Figure 1is an example of the first few levels of a Hofbauer tower. The symmetric tent map $T_{F}$ associated with this Hofbauer tower is called the map with Fibonacci combinatorics; that is, the map $T_{F}$ is defined such that

$$
S_{0}=1, S_{1}=2, S_{2}=3, S_{3}=5, S_{4}=8, S_{5}=13, \ldots
$$

We note that the kneading map for $T_{F}$ is defined by $Q_{T_{F}}(k)=k-2$ and that the parameter value for $T_{F}$ is given by $F \approx 1.7292119317$.

We now prove several results about Hofbauer towers and omega-limit sets. We begin by stating the following lemma.

Lemma 3.1 ([8, Lemma 2.1]). Let $f$ be a unimodal map (with no wandering intervals and no attracting periodic orbits) and suppose $\lim _{k \rightarrow \infty} Q_{f}(k)=\infty$. Then $\lim _{n \rightarrow \infty}\left|D_{n}\right|=0$.

Proposition 3.2 ([2, Proposition 2.3]). Let $f$ be a unimodal map and suppose $\lim _{k \rightarrow \infty} Q_{f}(k)=\infty$. Then for each $x \in \omega(c), x$ lies in infinitely many levels $D_{n}$ of the Hofbauer tower.

Proof. The following proof is similar to the proof of [8, Lemma 2.1]. Fix $K \in \mathbb{N}$. We denote each level $D_{n}$ of the Hofbauer tower by $D_{n}=\left[c_{\beta(n)} ; c_{n}\right]$. For all $m<S_{K}$, set $L_{m} \geq S_{K}$ to be such that $\beta\left(L_{m}\right)=m$ and $D_{L_{m}}$ is the largest interval of all the $D_{n}$ 's with $n \geq S_{K}$ and $\beta(n)=m$; we know such an $L_{m}$ exists by Lemma 3.1.

If $n \geq S_{K}$, then there exists a nested sequence of intervals $D_{n} \subset D_{\beta(n)} \subset \cdots \subset$ $D_{\beta^{r}(n)}$, where $r$ is the least integer such that $\beta^{r}(n)<S_{K}$; here, for example, $\beta^{2}(n)=\beta(\beta(n))$. We set $m=\beta^{r}(n)$. Then $D_{n} \subset D_{\beta^{r-1}(n)} \subseteq D_{L_{m}}$. Thus $\omega(c) \subset \overline{\bigcup_{n \geq S_{k}} D_{n}} \subset \overline{\bigcup_{m<S_{K}} D_{L_{m}}}=\bigcup_{m<S_{K}} D_{L_{m}}$. Hence for all $x \in \omega(c)$ and $K \in \mathbb{N}$, there exists an $n \geq S_{K}$ such that $x \in D_{n}$ (namely, $n=L_{m}$ for some $\left.m<S_{k}\right)$.

Lemma 3.3. Let $f$ be a unimodal map with $\lim _{k \rightarrow \infty} Q_{f}(k)=\infty$. Suppose $x \in \omega(c)$ has only one preimage lying in $\omega(c)$. Then there exists an $N$ such that whenever $x \in D_{n}$ for $n \geq N$ and $y \in D_{n-1}$ with $f(y)=x$, then $y \in \omega(c)$. 


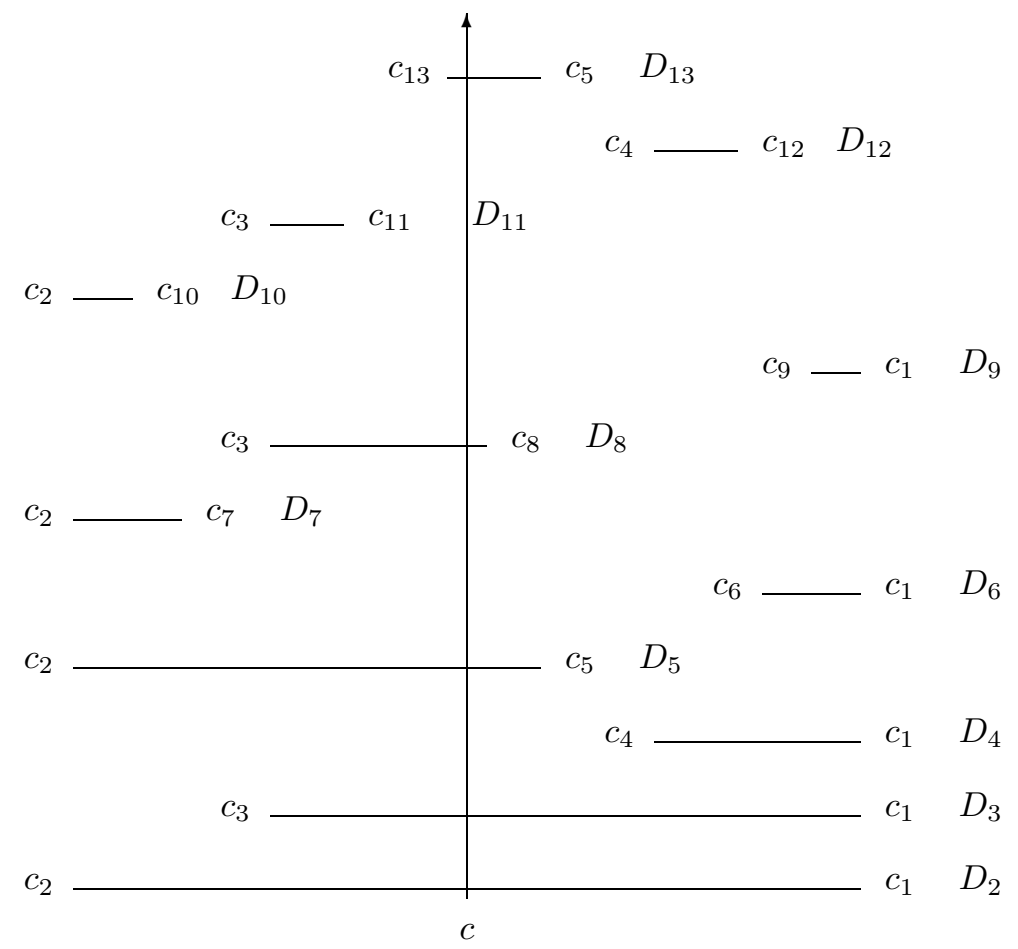

FiguRE 1. Hofbauer tower for Fibonacci combinatorics

Proof. Suppose $x \in \omega(c)$ has a unique preimage in $\omega(c)$. As $x \in\left[c_{2}, c_{1}\right]$, if $x \neq c_{1}$, then $x$ has two preimages in $[0,1], y$ and $\hat{y}$. Without loss of generality, we assume $y<c<\hat{y}$. If $y<c_{2}$, then for all $n \geq 3$, if $x \in D_{n}$, then $y \notin D_{n-1}$, but $\hat{y} \in D_{n-1}$. As $x \in \omega(c)$ and $\left.f\right|_{\omega(c)}$ is an onto mapping with $\omega(c) \subset\left[c_{2}, c_{1}\right]$, it follows that $\hat{y} \in \omega(c)$. Thus assume $y \geq c_{2}$. Hence $y, \hat{y} \in\left[c_{2}, c_{1}\right]$. Suppose $y$ lies in infinitely many levels of the Hofbauer tower for $f$. We define an increasing sequence $\left\{n_{k}\right\}$ such that $y \in D_{n_{k}}$ for all $k \in \mathbb{N}$. As $\lim _{k \rightarrow \infty} Q_{f}(k)=\infty$ and $\left|D_{n}\right| \rightarrow 0$, it follows that $\lim _{k \rightarrow \infty} c_{n_{k}}=y$, and hence $y \in \omega(c)$. Similarly, if $\hat{y}$ lies in infinitely many levels of the Hofbauer tower for $f$, then $\hat{y} \in \omega(c)$. As $x$ has a unique preimage in $\omega(c)$, it follows that only one of $y$ and $\hat{y}$ can lie in infinitely many levels of the Hofbauer tower, and thus either $y$ or $\hat{y}$ will be the unique preimage of $x$ in $\omega(c)$. Hence the result holds.

Lemma 3.4. Let $f$ be a unimodal map with $\lim _{k \rightarrow \infty} Q_{f}(k)=\infty$ and suppose $x \in \omega(c)$ with $x \neq c_{i}$ for all $i \geq 0$. If we set $\left\{n_{k}\right\}$ to be an increasing sequence of integers such that $x \in D_{n_{k}}$ for all $k \in \mathbb{N}$, then $\lim _{k \rightarrow \infty} \beta\left(n_{k}\right)=\infty$.

Proof. Let $x \in \omega(c)$ as above and suppose $\left\{n_{k}\right\}_{k \geq 1}$ is an increasing sequence such that $x \in D_{n_{k}}$ for all $k \in \mathbb{N}$. Suppose that $\lim _{k \rightarrow \infty} \beta\left(n_{k}\right) \neq \infty$. Then there exists an $N \in \mathbb{N}$ such that $\beta\left(n_{k}\right) \leq N$ for infinitely many $k \in \mathbb{N}$. Let $d=\min \left\{\left|x-c_{i}\right|\right\}_{i=1}^{N}$. As $D_{n_{k}}=\left[c_{n_{k}} ; c_{\beta\left(n_{k}\right)}\right]$ and $\beta\left(n_{k}\right) \leq N$ for infinitely many $k$, it follows that $\left|D_{n_{k}}\right|>d$ for infinitely many $k \in \mathbb{N}$. This contradicts Lemma 3.1, and hence the result holds. 
We now use the Hofbauer tower to investigate the collection of endpoints of the inverse limit space $(I, f)$ where the single bonding map $f$ is unimodal with $\lim _{k \rightarrow \infty} Q_{f}(k)=\infty$.

\section{INVERSE Limit SPACES When $\left.f\right|_{\omega(c)}$ IS (NEARLY) ONE-TO-ONE}

Earlier work (see, for example, [1, 2]) showed that in the case where $f \in \mathcal{A}$ and $\lim _{k \rightarrow \infty} Q_{f}(k)=\infty$, the collection of endpoints of the inverse limit space $(I, f)$ is precisely $\mathcal{E}$. When $f \in \mathcal{A}$ is chosen such that $\lim _{k \rightarrow \infty} Q_{f}(k) \neq \infty$, there exist examples where $\mathcal{E}$ is precisely the collection of endpoints of $(I, f)$ and examples where $\mathcal{E}$ properly contains the collection of endpoints. Whenever $f \in \mathcal{A}$, recall that $\left.f\right|_{\omega(c)}$ is one-to-one. We now restrict ourselves to unimodal maps $f$ where $\lim _{k \rightarrow \infty} Q_{f}(k)=\infty$ and assume only that $\left.f\right|_{\omega(c)}$ is one-to-one, a weaker condition than $f \in \mathcal{A}$.

We are now ready to prove the main result of this paper. Given a point $x=$ $\left(x_{0}, x_{1}, x_{2}, \ldots\right) \in \mathcal{E}_{f}$, we look at specific levels of the Hofbauer tower associated with the unimodal map $f$; these levels are each chosen to contain a coordinate $x_{i}$ of $x$. We then use the structure of the Hofbauer tower to identify whether $x$ is an endpoint of $(I, f)$.

Theorem 4.1. Suppose $f$ is a unimodal map, $\lim _{k \rightarrow \infty} Q_{f}(k)=\infty$, and $\left.f\right|_{\omega(c)}$ is one-to-one. Then $\mathcal{E}$ is precisely the collection of endpoints of $(I, f)$.

Proof. Without loss of generality, let $x=\left(x_{0}, x_{1}, x_{2}, \ldots\right) \in \mathcal{E}$ be such that $x_{i} \neq c$ for all $i \geq 0$. As $x_{0} \in \omega(c)$, Proposition 3.2 guarantees an increasing sequence $\left\{n_{k}\right\}$ such that $x_{0} \in D_{n_{k}}$ for all $k \in \mathbb{N}$. Let $N$ be chosen as in Lemma 3.3. we note that $\left\{n_{k}\right\}_{k \geq 1}$ may be chosen such that $n_{1}>S_{l}>N$, where $S_{l}$ is the first cutting time after $N$. Then $\mathcal{I}_{\beta\left(n_{k}\right)-1}(x) \cdots \mathcal{I}_{1}(x)=e_{1} \cdots e_{\beta\left(n_{k}\right)-1}$ for all $k \in \mathbb{N}$. As $\lim _{k \rightarrow \infty} \beta\left(n_{k}\right)=\infty$ (by Lemma 3.4), it follows that one of $\tau_{R}(x)$ or $\tau_{L}(x)$ is infinite. Without loss of generality, we assume $\tau_{R}(x)=\infty$. If $x_{0}=\sup \pi_{0}(\Gamma(x))$, then by Proposition 2.2, $x$ is an endpoint of $(I, f)$. We thus suppose $x_{0} \neq \sup \pi_{0}(\Gamma(x))$, and thus $x_{0}<\sup \pi_{0}(\Gamma(x))$. Recall $\sup \pi_{0}(\Gamma(x))=\inf \left\{c_{n} \mid \mathcal{I}_{n-1}(x) \cdots \mathcal{I}_{1}(x)=\right.$ $e_{1} \cdots e_{n-1}$ and $e_{1} \cdots e_{n-1}$ has even parity $\}$ by Lemma 2.3 .

Let $d_{1}=\left|x_{0}-\sup \pi_{0}(\Gamma(x))\right|>0$. By Lemma 3.1 there exists a $K$ such that for all $k \geq K,\left|D_{n_{k}}\right|<d_{1}$; as $x_{0}, c_{\beta\left(n_{k}\right)} \in D_{n_{k}}$, it follows that $c_{\beta\left(n_{k}\right)}<\sup \pi_{0}(\Gamma(x))$ for all $k \geq K$. If $e_{1} \cdots e_{\beta\left(n_{k}\right)-1}$ has even parity, then by Lemma 2.3 we would have $c_{\beta\left(n_{k}\right)}<$ $\inf \left\{c_{n} \mid \mathcal{I}_{n-1}(x) \mathcal{I}_{n-2}(x) \cdots I_{1}(x)=e_{1} e_{2} \cdots e_{n-1}\right.$ and $e_{1} e_{2} \cdots e_{n-1}$ has even parity $\}$; $c_{\beta\left(n_{k}\right)}$ would both belong to this set and be less than its infinum, a contradiction. Hence it follows that $e_{1} \cdots e_{\beta\left(n_{k}\right)-1}$ has odd parity for all $k \geq K$.

Because $\mathcal{I}_{\beta\left(n_{k}\right)-1}(x) \cdots \mathcal{I}_{1}(x)=e_{1} \cdots e_{\beta\left(n_{k}\right)-1}$ for all $k \geq K$ and the $\lim _{k \rightarrow \infty} \beta\left(n_{k}\right)$ $=\infty$, it follows that $\tau_{L}(x)=\infty$. If $x_{0}=\inf \pi_{0}(\Gamma(x))$, then $x$ is an endpoint of $(I, f)$. We thus assume $x_{0} \neq \inf \pi_{0}(\Gamma(x))=\sup \left\{c_{n} \mid \mathcal{I}_{n-1}(x) \cdots \mathcal{I}_{1}(x)=\right.$ $e_{1} \cdots e_{n-1}$ and $e_{1} \cdots e_{n-1}$ has odd parity $\}$, in which case $x_{0}>\inf \pi_{0}(\Gamma(x))$. Let $d_{2}=\left|x_{0}-\inf \pi_{0}(\Gamma(x))\right|>0$ and fix $M>K$ such that $\left|D_{n_{m}}\right|<d_{2}$ for all $m \geq M$. As $x_{0}, c_{\beta\left(n_{m}\right)} \in D_{n_{m}}$ for all $m \geq M$, it follows that $c_{\beta\left(n_{m}\right)}>\inf \pi_{0}(\Gamma(x))$, a contradiction. Hence $x$ is an endpoint of $(I, f)$.

The following definition allows us to expand Theorem 4.1 to a larger class of unimodal maps. 
Definition 4.2. We call a map nearly one-to-one on its omega-limit set if the set of points for which $\left.f\right|_{\omega(c)}$ is not one-to-one is finite and non-empty; i.e., $\{y \mid$ $y \in \omega(c)$ and there exists $z_{1} \neq z_{2} \in \omega(c)$ with $\left.f\left(z_{1}\right)=f\left(z_{2}\right)=y\right\}$ is finite and non-empty.

Corollary 4.3. Let $f$ be a unimodal map such that $\left.f\right|_{\omega(c)}$ is nearly one-to-one on $\omega(c)$ and suppose further that $\lim _{k \rightarrow \infty} Q_{f}(k)=\infty$. Then $\mathcal{E}$ is precisely the collection of endpoints of $(I, f)$.

Proof. Let $x \in \mathcal{E}$. As $\lim _{k \rightarrow \infty} Q_{f}(k)=\infty$, it follows that $c$ is not periodic and $\omega(c)$ is a minimal Cantor set. As the collection of points in $\omega(c)$ with two preimages lying in $\omega(c)$ is finite, the number of positions $x_{i}$ in $x$ for which $x_{i}$ has two preimages in $\omega(c)$ is finite. If not, then $x_{j}=x_{i}$ for some $i \neq j$, but this violates the minimality of $\omega(c)$. Let $N$ be the largest index for which $x_{N}$ has two preimages in $\omega(c)$. Then $\hat{f}^{-(N+1)}(x)$ is such that every coordinate has a unique preimage in $\omega(c)$. Now apply a proof similar to the proof of Theorem 4.1

Remark 4.4. Consider $T_{F}$, the symmetric tent map with Fibonacci combinatorics. Every point in $\omega(c) \backslash\{c\}$ has a unique preimage in $\omega(c)$, whereas $c$ has two preimages in $\omega(c)$. Hence $T_{F}$ is nearly one-to-one on $\omega(c)$, and by Corollary 4.3, it follows that the collection of endpoints of $\left(I, T_{F}\right)$ is exactly $\mathcal{E}$.

We now make some observations about the set of endpoints of an inverse limit space $(I, f)$ and the set $\mathcal{E}$.

Proposition 4.5. Let $f$ be a unimodal map. Then $\mathcal{E}$ is closed.

Proof. Let $\left\{x^{i}\right\}_{i \geq 0}=\left\{\left(x_{0}^{i}, x_{1}^{i}, x_{2}^{i}, \ldots\right)\right\}_{i \geq 0}$ be a sequence of points in $\mathcal{E}$ such that $\lim _{i \rightarrow \infty} x^{i}=z$ for some $z \in(I, f)$. As $\lim _{i \rightarrow \infty} x^{i}=z$, for each $\epsilon>0$ there exists an $N \in \mathbb{N}$ such that $d\left(x^{n}, z\right)<\epsilon$ for all $n \geq N$. Hence, for all $j \in \mathbb{N}$ there exists an $N_{j} \in \mathbb{N}$ such that $\pi_{l}\left(x^{n}\right)=z_{l}$ for all $l \leq j$ and $n \geq N_{j}$. Hence $\lim _{n \rightarrow \infty} \pi_{l}\left(x^{n}\right)=z_{l}$ for all $l \in \mathbb{N}$. As $\pi_{l}\left(x^{n}\right) \in \omega(c)$ for all $l, n \in \mathbb{N}$, it follows that $z_{l} \in \omega(c)$ for all $l \in \mathbb{N}$. Hence $z \in \mathcal{E}$.

Proposition 4.6. Suppose $\left.f\right|_{\omega(c)}$ is one-to-one, $\omega(c)$ is a minimal set, and $\mathcal{E}$ properly contains the collection of endpoints of $(I, f)$. Then the collection of endpoints of $(I, f)$ is not a closed set.

Proof. Let $x=\left(x_{0}, x_{1}, x_{2}, \ldots\right) \in \mathcal{E}$ be an endpoint of $(I, f)$ and suppose $y=$ $\left(y_{0}, y_{1}, y_{2}, \ldots\right) \in \mathcal{E}$ is a non-endpoint. As $\omega(c)$ is minimal, $y_{0} \in \omega(c)=\omega\left(x_{0}\right)$. Thus there exists some increasing sequence $\left\{k_{i}\right\}_{i \geq 0}$ such that $\lim _{i \rightarrow \infty} f^{k_{i}}\left(x_{0}\right)=$ $y_{0}$. As $f$ is a homeomorphism on $\omega(c)$, it follows that $\lim _{i \rightarrow \infty} f^{-j}\left(f^{k_{i}}\left(x_{0}\right)\right)=$ $\lim _{i \rightarrow \infty} f^{k_{i}-j}\left(x_{0}\right)=f^{-j}\left(y_{0}\right)$ for all $j \geq 0$. As $f^{k_{i}-j}\left(x_{0}\right)$ is unique in $\omega(c)$ for all $i, j \in \mathbb{N}$ and $f^{-j}\left(y_{0}\right)=y_{j}$, it follows that $\lim _{i \rightarrow \infty} \hat{f}^{k_{i}}(x)=y \in \mathcal{E}$. As each $\hat{f}^{k_{i}}(x)$ is an endpoint of $(I, f)$ and $y$ is not an endpoint of $(I, f)$, it follows that the collection of endpoints of $(I, f)$ is not a closed set.

Corollary 4.7. Let $f$ and $g$ be unimodal maps such that $\left.g\right|_{\omega(c)}$ is one-to-one. Suppose further that $\mathcal{E}_{f}$ is precisely the collection of endpoints of $(I, f)$ and $\mathcal{E}_{g}$ properly contains the endpoints of $(I, g)$. Then $(I, f)$ and $(I, g)$ are not homeomorphic.

Proof. This follows immediately from Propositions 4.5 and 4.6. 
We would like to be able to use easily identifiable structures within inverse limit spaces, such as endpoints, to clearly distinguish between the inverse limit spaces generated by two distinct unimodal bonding maps. It is known that given two distinct symmetric tent maps $f$ and $g$, the inverse limit spaces $(I, f)$ and $(I, g)$ are not homeomorphic [3, but the actual topological structures of these inverse limit spaces are not completely understood. We hope that by fully understanding the behavior of the collection of endpoints in a given inverse limit space we will better understand its topological structure. The question remains whether one can use only structures such as endpoints to distinguish between two inverse limit spaces. Further, if two unimodal maps $f$ and $g$ are given such that the collection of endpoints of $(I, f)$ and $(I, g)$ are $\mathcal{E}_{f}$ and $\mathcal{E}_{g}$, respectively, can the collection of endpoints be used to distinguish $(I, f)$ from $(I, g)$ ? If not, what other combinatoric tools, such as kneading maps, must be used?

The results here rely heavily on $\left.f\right|_{\omega(c)}$ being one-to-one and $\lim _{k \rightarrow \infty} Q_{f}(k)=\infty$. We do not have any examples where $\lim _{k \rightarrow \infty} Q_{f}(k)=\infty$ and $\mathcal{E}$ is not the collection of endpoints of $(I, f)$. Is it true that any unimodal map $f$ with $\lim _{k \rightarrow \infty} Q_{f}(k)=\infty$ will be such that $\mathcal{E}$ is exactly the collection of endpoints of $(I, f)$, regardless of the behavior of $\left.f\right|_{\omega(c)}$ ? Further, can the techniques in this paper be extended to identify the collection of endpoints of $(I, f)$ when $\lim _{k \rightarrow \infty} Q_{f}(k)=\infty$ but $\left.f\right|_{\omega(c)}$ is not (nearly) one-to-one?

\section{REFERENCES}

[1] L. Alvin and K. Brucks, Adding machines, endpoints, and inverse limit spaces, Fund. Math. 209 (2010), 81-93. MR2652593 (2011f:37029)

[2] L. Alvin and K. Brucks, Adding machines, kneading maps, and endpoints, Topology Appl. 158 (2011), 542-550. MR2754376 (2012e:37076)

[3] M. Barge, H. Bruin, and S. Stimac, The Ingram conjecture, preprint.

[4] M. Barge and J. Martin, Endpoints of inverse limit spaces and dynamics, Lecture Notes in Pure and Appl. Math. 170 (1995), 165-182. MR1326840(96b:54062)

[5] L. Block, J. Keesling, and M. Misiurewicz, Strange adding machines, Ergod. Th. \& Dynam. Sys. 26 (2006), 673-682. MR2237463(2007c:37037)

[6] K.M. Brucks and H. Bruin, Topics from one-dimensional dynamics, Cambridge University Press (2004). MR2080037 (2005j:37048)

[7] H. Bruin, Planar embeddings of inverse limit spaces of unimodal maps, Topology Appl. 96 (1999), 191-208. MR 1709688 (2000g:37010)

[8] H. Bruin, (Non)invertibility of Fibonacci-like unimodal maps restricted to their critical omega-limit sets, Topology Proc. 37 (2011), 459-480. MR2746141 (2011m:37062)

[9] P. Collet and J-P. Eckmann, Iterated maps on the interval as dynamical systems, Birkhäuser (1980). MR613981 (82j:58078)

[10] W. de Melo and S. van Strien, One-dimensional dynamics, Springer Verlag (1993). MR.1239171 (95a:58035)

[11] F. Hofbauer and G. Keller, Quadratic maps without asymptotic measure, Commun. Math. Phys. 127 (1990), no. 2, 319-337. MR1037108 (91a:58103)

Department of Mathematics and Statistics, University of West Florida, 11000 University Parkway, Pensacola, Florida 32514

E-mail address: lalvin@uwf.edu 